\title{
Dynamical Landscape of Heart Rhythm in Discerning Erratic Rhythm in Elderly People
}

\author{
D Makowiec ${ }^{1}$, J Wdowczyk ${ }^{2}$, D Wejer ${ }^{3}$, M Żarczyńska-Buchowiecka ${ }^{2}$, ZR Struzik ${ }^{4,5}$ \\ ${ }^{1}$ Institute of Theoretical Physics and Astrophysics, University of Gdańsk, Poland \\ ${ }^{2}$ First Cardiology Clinic, Medical University of Gdańsk, Poland \\ ${ }^{3}$ Institute of Experimental Physics, University of Gdańsk, Poland \\ ${ }^{4}$ RIKEN Brain Science Institute, Japan \\ ${ }^{5}$ Graduate School of Education, University of Tokyo, Japan
}

\begin{abstract}
We hypothesize that increase with age of short-term measures of heart rhythm [a way in which accelerations and decelerations appear], especially dynamical pattern indices [Transition Rates $\left(S_{T}\right)$ or self-Transfer Entropy $(s T E)]$, provide indicates for unhealthy autonomic activity or cardiac tissue remodeling in elderly people. Hence they can help in early recognition of arrhythmogenesis processes.

Based on properties of heart rhythm of 190 healthy persons, grouped into their age decade, we have found that both $S_{T}$ and sTE are efficient separators for discerning elderly people with erratic rhythm. The values in minimum in $S_{T}=2.4$ and minimum in sTE $=0.27$, obtained by square function approximation, have been used to divide subjects of 70s and 80s into two groups. For all entropic measures these groups are different $(p<0.001$, in t-test or Man-Whitney in case normality test failed). The fragmentation metrics [based on statistics of signs of heart rate changes] PPP and PAS also distingushed these groups though at greater p-value, and PSS yielded that the groups are identical. The minima of these function did not give satisfactory division of elderly into groups.

Concluding, frequent changes in heart rate acceleration sign and size are the best signature for anomalous levels of short-term heart rate variability in elderly people.
\end{abstract}

\section{Introduction}

The healthy human heart remains under the permanent influence of both branches of the autonomic neural system (ANS): the parasympathetic (considered to slow down heart rate) and the sympathetic (considered to speed up heart rate). Many measures estimating heart rate variability (HRV) have been proposed in order to quantify the regulatory function of the ANS, see [1-3]. Intensive healthy population studies have found a correlation between an increase in age and a decrease in many HRV indices, including indices such as RMSDD, pNN50 and HF, which are known to describe the short-term HRV (ST-HRV) [4-10]. Therefore, higher values of HRV have been attributed to better organization of feedback reflexes driving the organism's response to actual body needs. However, there are observations suggesting that abnormal levels of short-term HRV indices should be related to erratic rhythms, i.e., rhythms resulting from remodeling of the cardiac tissue due to disease or aging [10-13].

In the following, we hypothesize that increase of shortterm measures of dynamical patterns in elderly provides indicates of unhealthy autonomic activity, or possible erratic rhythm resulting from degradation of cardiac tissue, or both. Such erratic rhythms might be the first stage of silently developing arrhythmogenesis.

The term "dynamical landscape of heart rhythm" was proposed in [14] to collect HRV indices focused on quantifying differences in the subsequent heart periods: decelerations and accelerations, the so-called RR-increments. Standard ST-HRV indices: RMSDD, pNN50 and HF are also considered to belong to the set of the dynamical landscape measures.

Erratic rhythms are episodic and often develop when the heart slows down, as it naturally happens during the nocturnal rest. Therefore long series of nocturnal recordings are investigated by us.

\section{Methods}

Twenty-four hour ECG Holter signals were recorded from 190 healthy participants. All of them gave the written permission of informed consent which received approval by the Ethic Committee of Medical University of Gdansk. The participants were grouped according to their age decade: 20's (35 subjects: 18 women), 30's (23sub- 
jects: 11 women), 40's (33 subjects: 13 women), 50's (31 subjects: 13 women), 60 's (27 subjects: 12 women), 70's (22 subjects: 10 women), 80's (18 subjects: 11 women).

The signals were analyzed on a Del Mar Reynolds system. The sampling rate of ECG was $128 \mathrm{~Hz}$ which ensured $8 \mathrm{~ms}$ accuracy for time identification of R-peaks in QRScomplexes. Quality of ECG recordings and accuracy of R-peak detection were verified by visual inspection by experienced cardiologists. All normal beats were carefully annotated, so that only sinus rhythms were considered.

The period of nocturnal rest was assigned individually, in each recording separately, according to the appearance of consecutive hours with the low rate. The non-normal beats were edited (replaced by the median of the last 7 normal beats). For each person 20000 subsequent normalto-normal RR-intervals were extracted.

We used metrics which are based on probability that particular RR-increments

$$
\delta R R(i)=R R(i)-R R(i-1) \text { for } i=1,2, \ldots, N
$$

occur in a signal. Subsequently, probability for two-event patterns $(\delta R R(i), \delta R R(i+1))$ and three-event patterns $(\delta R R(i), \delta R R(i+1), \delta R R(i+2))$ is also estimated.

For detail description of indices used we refer to [14]. Here we only provide their brief description to introduce the notation:

$-\Delta_{I} \in\left\{\Delta_{\min } \ldots, 0, \ldots, \Delta_{\max }\right\}$ is any RR-increment ; $-p()$ denotes probability of events enclosed in brackets ();

$-\operatorname{ShEn} 1=-\sum_{I} p\left(\Delta_{I}\right) \ln p\left(\Delta_{I}\right)$;

- ShEn2 $=-\sum_{I, J} p\left(\Delta_{I}, \Delta_{J}\right) \ln p\left(\Delta_{I}, \Delta_{J}\right)$ for a pair $\left(\Delta_{I}, \Delta_{J}\right)$ of consecutive in time events;

- ShEn3 = - $\sum_{I, J, K} p\left(\Delta_{I}, \Delta_{J}, \Delta_{K}\right) \ln p\left(\Delta_{I}, \Delta_{J}, \Delta_{K}\right)$ for a triple $\left(\Delta_{I}, \Delta_{J}, \Delta_{K}\right)$ of consecutive in time events;

$-\mathrm{S}_{T}=\mathrm{ShEn} 2-\mathrm{ShEn} 1$

$-\mathrm{sTE}=(\operatorname{ShEn} 2-\mathrm{ShEn} 1)-(\operatorname{ShEn} 3-\mathrm{ShEn} 2)$;

- a: any acceleration: $\Delta_{I}<0$;

- d: any deceleration: $\Delta_{I}>0$;

- PIP $=\sum_{a d} p(a d)+\sum_{d a} p(d a):$ probability of abrupt changes in the sign of two subsequent RR-increments - PSS $=1-\left[\sum_{a a a} p(a a a)+\sum_{d d d} p(d d d)\right]$ : complement to the probability of short monotonic accelerations or decelerations;

- PAS $=\sum_{a d a} p(a d a)+\sum_{d a d} p(d a d):$ probability of alternative three-event patterns.

As the qualification whether $\delta R R(i) \neq 0$, hence if $\delta R R(i)$ is an acceleration or deceleration, depends on signal resolution, we performed estimates for the resolution of a signal, i.e., with $8 \mathrm{~ms}$ and with doubled resolution of $16 \mathrm{~ms}$.

Changes in a short-term index are often described as being of letter $J$ shape. Therefore, we used a square function approximation to find the index dependence on age decade. The nonlinear regression provided us with values which then allowed us to discern elderly people not affected by erratic rhythms (called OK group) and people with erratic rhythms (called NOK group). For these groups we present the mean of the tensor of self-transfer entropy, for details see [15], estimated as follows

$$
\begin{array}{r}
\operatorname{TsTE}\left(\Delta_{J}, \Delta_{K}\right)=p\left(\Delta_{K}\right) \ln p\left(\Delta_{K}\right) \\
-2 p\left(\Delta_{J}, \Delta_{K}\right) \ln p\left(\Delta_{J}, \Delta_{K}\right) \\
+\sum_{\Delta_{I}} p\left(\Delta_{I}, \Delta_{J}, \Delta_{K}\right) \ln p\left(\Delta_{I}, \Delta_{J}, \Delta_{K}\right)
\end{array}
$$

All statistical tests and approximations have been performed with SigmaPlot13 software.

\section{Results}

The square function approximations were estimated for all the quantities listed above. However, only for $S_{T}$ and sTE these findings obtained the statistical significance. Namely the coefficient of determination how well the regression model describes the data was $R^{2}>0.3$. Similarly high though lower $R^{2}$ values were obtained for ShEn1,ShEn2, and ShEn3. For PIP, PSS, and PAS $R^{2} \approx$ 0.12 .
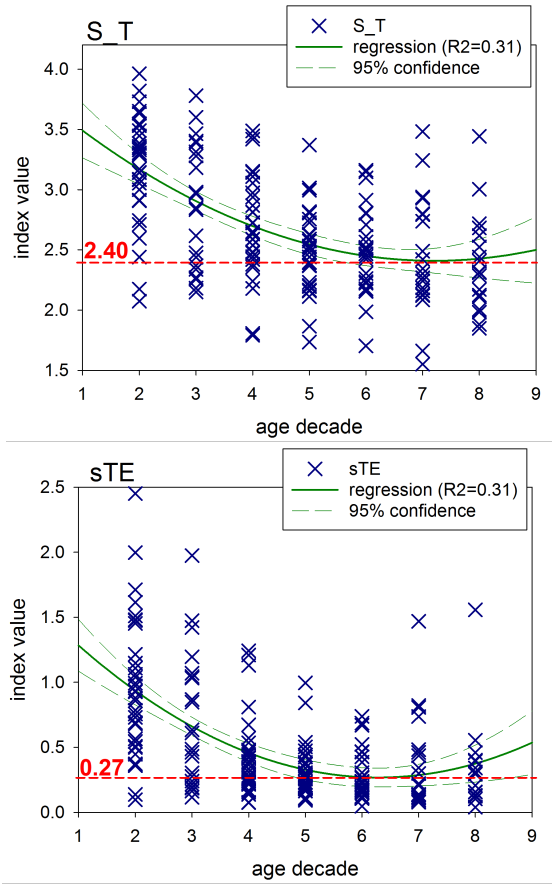

Figure 1. Results of $\mathbf{S}_{T}$ and sTE for all signals grouped in age decades are presented together with the square function regression. The minimal values in these approximations, namely 2.40 for $S_{T}$ at 70 's (the upper) panel and 0.27 for sTE at 60 's (the bottom panel) serve further as the group delimiters.

Fig. 1 presents the method in which the separators for the groups of elderly people OK and NOK were defined. 
Table 1. Central values (mean or median) and dispersion (SEM or first and third quartiles) in groups OK and NOK for dynamical landscape indices considered by us.

\begin{tabular}{|c|c|c|c|c|}
\hline $\begin{array}{l}\text { Index } \\
\text { name }\end{array}$ & $\begin{array}{l}\text { group } \\
\text { name }\end{array}$ & $\begin{array}{c}\text { mean or } \\
\text { median }\end{array}$ & $\begin{array}{l} \pm \text { SEM or } \\
\text { [,]quartiles }\end{array}$ & $\begin{array}{c}\text { OK vs NOK } \\
\text { p-value }\end{array}$ \\
\hline \multicolumn{5}{|c|}{ Shannon entropy based measures: } \\
\hline \multirow[t]{2}{*}{$\mathrm{S}_{\mathrm{T}}$} & OK & 2.10 & \pm 0.05 & $<0.001$ \\
\hline & NOK & 2.84 & \pm 0.08 & \\
\hline \multirow[t]{2}{*}{ sTE } & OK & 0.13 & {$[0.13,0.15]$} & $<0.001$ \\
\hline & NOK & 0.46 & {$[0.40,0.79]$} & \\
\hline \multirow[t]{2}{*}{ ShEn3 } & OK & 6.3 & \pm 0.14 & $<0.001$ \\
\hline & NOK & 8.2 & \pm 0.17 & \\
\hline \multirow[t]{2}{*}{ ShEn2 } & OK & 4.3 & \pm 0.10 & $<0.001$ \\
\hline & NOK & 6.0 & \pm 0.18 & \\
\hline \multirow[t]{2}{*}{ ShEn1 } & OK & 2.25 & {$[2.1,2.3]$} & $<0.001$ \\
\hline & NOK & 3.0 & {$[2.8,3.3]$} & \\
\hline \multicolumn{5}{|c|}{ fragmentation indices: } \\
\hline \multirow[t]{2}{*}{ PIP } & OK & 0.39 & \pm 0.012 & $<0.001$ \\
\hline & NOK & 0.50 & \pm 0.017 & \\
\hline \multirow[t]{2}{*}{ PAS } & $\mathrm{OK}$ & 0.177 & \pm 0.013 & 0.012 \\
\hline & NOK & 0.250 & \pm 0.019 & \\
\hline \multirow[t]{2}{*}{ PSS } & OK & 0.928 & {$[0.904,0.951]$} & NS \\
\hline & NOK & 0.933 & {$[0.908,0.960]$} & \\
\hline \multirow[t]{2}{*}{$\mathrm{p}(\mathrm{ad})$} & $\mathrm{OK}$ & 0.196 & \pm 0.007 & $<0.001$ \\
\hline & NOK & 0.251 & \pm 0.009 & \\
\hline \multirow[t]{2}{*}{$\mathrm{p}(\mathrm{da})$} & OK & 0.192 & \pm 0.006 & $<0.001$ \\
\hline & NOK & 0.251 & \pm 0.008 & \\
\hline \multirow[t]{2}{*}{$\mathrm{p}(\mathrm{aa})$} & $\mathrm{OK}$ & 0.122 & \pm 0.007 & 0.005 \\
\hline & NOK & 0.159 & \pm 0.009 & \\
\hline \multirow[t]{2}{*}{$\mathrm{p}(\mathrm{dd})$} & OK & 0.118 & \pm 0.008 & NS \\
\hline & NOK & 0.134 & \pm 0.009 & \\
\hline \multirow[t]{2}{*}{$\mathrm{p}(\mathrm{aaa})$} & OK & 0.038 & {$[0.022,0.046]$} & NS \\
\hline & NOK & 0.037 & {$[0.020,0.054]$} & \\
\hline \multirow[t]{2}{*}{$\mathrm{p}(\mathrm{ddd})$} & OK & 0.036 & \pm 0.004 & NS \\
\hline & NOK & 0.030 & \pm 0.005 & \\
\hline \multirow[t]{2}{*}{$\mathrm{p}(\mathrm{ada})$} & OK & 0.091 & \pm 0.007 & 0.020 \\
\hline & NOK & 0.131 & \pm 0.012 & \\
\hline \multirow[t]{2}{*}{$\mathrm{p}(\mathrm{dad})$} & OK & 0.087 & \pm 0.007 & 0.016 \\
\hline & NOK & 0.119 & \pm 0.009 & \\
\hline \multicolumn{5}{|c|}{ standard STV indices: } \\
\hline \multirow[t]{2}{*}{ RMSSD } & OK & 20.6 & {$[17.2,24.3]$} & $<0.001$ \\
\hline & NOK & 43.6 & {$[37.4,70.8]$} & \\
\hline \multirow[t]{2}{*}{ pNN50 } & OK & 1.21 & {$[0.67,2.1]$} & $<0.001$ \\
\hline & NOK & 18.0 & {$[11.4,28.4]$} & \\
\hline \multirow[t]{2}{*}{$\mathrm{HF}$} & OK & 96.6 & {$[67.3,131.4]$} & $<0.001$ \\
\hline & NOK & 462.6 & {$[318,1113]$} & \\
\hline \multicolumn{5}{|c|}{ Other HRV indices: } \\
\hline mean(RR) & OK & 870 & \pm 14 & 0.002 \\
\hline & NOK & 976 & \pm 23 & \\
\hline SDNN & OK & 67.2 & {$[58.7,80.5]$} & NS \\
\hline & NOK & 78.8 & {$[66.8,112.3]$} & \\
\hline
\end{tabular}
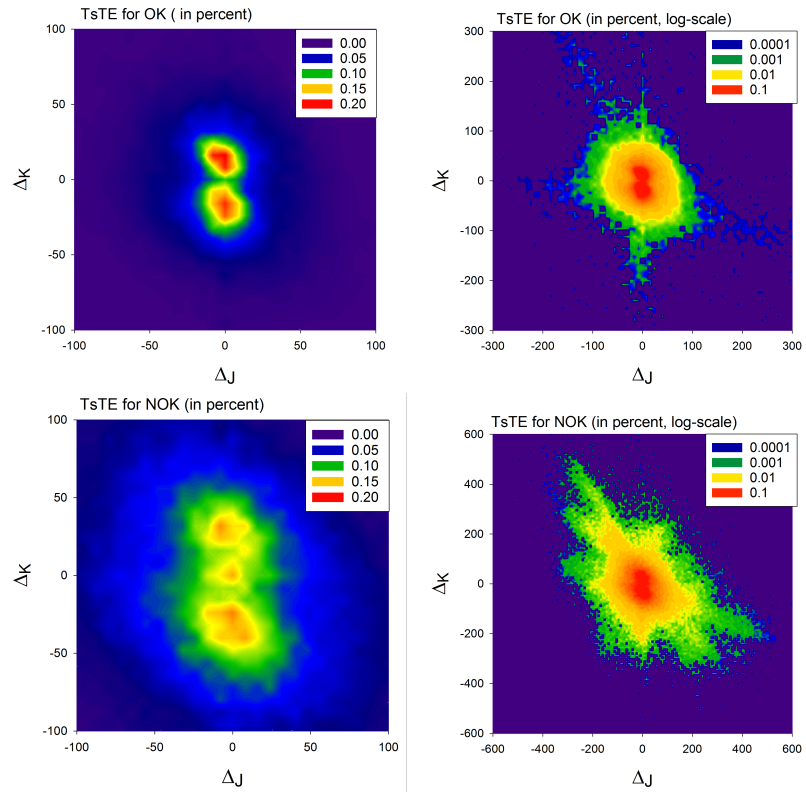

Figure 3. The mean tensor of self-transfer entropy (TsTE) for the considered groups OK and NOK. The left column is to show the main part of the tensors, the right columns shows rare events thanks to log-scale.

Then in Fig. 2 we show how these two separators, 2.40 for $\mathrm{S}_{T}$ and 0.27 for sTE, are related with each other for a given person. Consequently, the group OK consists of rhythms for which both indices are smaller than the minima (20 signals), and the group NOK contains signals for which both indices are greater than the minima (16 signals). The remaining four signals form a group of unclassified signals.

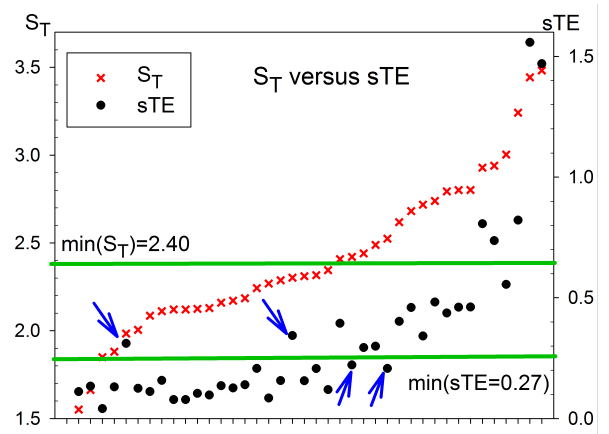

Figure 2. The group membership for signals of people in the age of 70's and 80's. By arrows four signals of the group unclassified are marked.

Then, in Table 1 the description of values for short-term HRV indices obtained for these groups is given. Together the result of the statistical test for difference between the groups OK and NOK is shown. It is noticeable that statisti- 
cal significance $(p<0.02)$ was attributed to the difference between unclassified signals and NOK for entropies ShEn2 and ShEn3 and for $\mathrm{S}_{T}$.

The analogical estimates were performed for a signal resolution of $16 \mathrm{~ms}$. All the results obtained, though quantitatively different, were qualitatively in agreement with the results obtained for resolution of $8 \mathrm{~ms}$.

Finally, in Fig. 3 we show the mean tensors of selftransfer entropy, see Eq. (1), calculated from tensors obtained for signals classified as $\mathrm{OK}$ and NOK. Notice that the contour plots are displayed in different ranges of $\left(\Delta_{J}, \Delta_{K}\right)$ events. Each group results are presented twice: by values scaled linearly to concentrate on properties of core dynamics, and by logarithms of values to see the whole variety of events.

\section{Discussion and summary}

Frequent changes in heart rate increments sign and size have proven to be the best signature for anomalous levels of ST-HRV in elderly people. The best discerning ability have been obtained for transition rates and self-transfer entropy. Perhaps it is because both measures are built on the concept of Shannon entropy. Shannon entropy quantifies the signal values following the logarithm of signal probability distribution function. So the entropy extracts and collects information across the scales in which the phenomenon operates. This way it enhances diversity in the probability distribution function. Fragmentation indices directly summarize probabilities of specially grouped dynamical patterns. The entropy based measures, while cumulating probabilities of events, scale them what provides evaluation both strength of given patterns and the pattern diversity.

\section{References}

[1] TaskForce. Task force of the European Society of Cardiology the North American Society of Pacing. Heart rate variability: standards of measurement, physiological interpretation, and clinical use. Circulation 1996;93(5):1043-1065.

[2] Goldberger AL, Stein PK. Evaluation of heart rate variability. http://www.uptodate.com/contents/evaluation-of-heartrate-variability, Access on July 15,2017 2015.

[3] Sassi R, Cerutti S, Lombardi F, Malik M, Huikuri HV, Peng CK, Schmidt G, Yamamoto Y, Gorenek B, Lip GH, Grassi G, Kudaiberdieva G, Fisher JP, Zabel M, Macfadyen R. Advances in heart rate variability signal analysis: joint position statement by the e-cardiology ESC working group and the European Heart Rhythm Association co-endorsed by the Asia Pacific Heart Rhythm Society. Europace 2015; 17:1341-1353. ISSN 1099-5129.

[4] Reardon M, Malik M. Changes in heart rate variability with age. Pacing and Clinical Electrophysiology 1996; 19(11):1863-1866. ISSN 1540-8159.

[5] Umetani K, Singer DH, McCraty R, Atkinson M. Twentyfour hour time domain heart rate variability and heart rate: relations to age and gender over nine decades. Journal of the American College of Cardiology 1998;31(3):593 - 601. ISSN 0735-1097.

[6] Pikkujämsä SM, Mäkikallio TH, Sourander LB, Räihä IJ, Puukka P, Skyttä J, Peng CK, Goldberger AL, Huikuri HV. Cardiac interbeat interval dynamics from childhood to senescence: comparison of conventional and new measures based on fractals and chaos theory. Circulation 1999; 100(4):393-399.

[7] Stein PK, Barzilay JI, Chaves PHM, Domitrovich PP, Gottdiener JS. Heart rate variability and its changes over 5 years in older adults. Age and Ageing 2009;38(2):212-218.

[8] Schumann AY, Bartsch RP, Penzel T, Ivanov PC, Kantelhardt JW. Aging effects on cardiac and respiratory dynamics in healthy subjects across sleep stages. Sleep 2010; 33(07):943-955.

[9] Makowiec D, Kaczkowska A, Wejer D, ŻarczyńskaBuchowiecka M, Struzik ZR. Entropic measures of complexity of short-term dynamics of nocturnal heartbeats in an aging population. Entropy 2015;17:1253-1272.

[10] Costa M, Davis R, Goldberger A. Heart rate fragmentation: A new approach to the analysis of cardiac interbeat interval dynamics. Frontiers in Physiology 2017;8:255. ISSN 1664 042X.

[11] Stein PK, Yanez D, Domitrovich PP, Gottdiener J, Chaves P, Kronmal R, Rautaharju P. Heart rate variability is confounded by the presence of erratic sinus rhythm. In Computers in Cardiology 2002, Memphis, USA. ISSN 02766547, Sept 2002; 669-672.

[12] Stein PK, Le Q, Domitrovich. Development of more erratic heart rate patterns is associated with mortality postmyocardial infarction. Journal of Electrocardiology 2008; 41(2):110-115.

[13] Nicolini P, Ciula MM, de Asmundus C, Magrini F, Brugada $\mathrm{P}$. The prognostic value of heart rate variability in the elderly, changing the perspective: from sympathovagal balance to chaos theory. PACE 2012;35(5):622-38.

[14] Wdowczyk J, Makowiec D, Gruchała M, Wejer D, Struzik ZR. Dynamical landscape of heart rhythm in longterm heart transplant recipients: A way to discern erratic rhythms. Frontiers in Physiology 2018;9:274. ISSN 1664042X.

[15] Makowiec D, Struzik ZR. Tensorial self-transfer entropy (tste) of rr heart interbeat signals and healthy ageing. In ESGCO2016 biological oscillations. 2016; 114-115.

Address for correspondence:

Danuta Makowiec

Institute of Theoretical Physics and Astrophysics of Gdansk University, 80-308 Gdańsk, ul. Wita Stwosza 57, Poland

e-mail: danuta.makowiec@ug.edu.pl 